\title{
Meeting report 8th Nottingham International Breast Cancer Conference,
Nottingham, UK, 16-19 September 2003
} Jane L Limer ${ }^{1}$, Alicia T Parkes ${ }^{1}$, Alison Waterworth ${ }^{1}$, Claire E Murphy ${ }^{1}$, Cathy R Tait ${ }^{1}$ and Caroline J Witton ${ }^{2}$

\author{
${ }^{1}$ Molecular Medicine Unit, St James's University Hospital, Leeds, UK \\ ${ }^{2}$ Division of Cancer Sciences and Molecular Pathology, Royal Infirmary, Glasgow, UK \\ Correspondence: Jane L Limer (e-mail: j.l.limer@leeds.ac.uk) \\ Published: 28 October 2003 \\ Breast Cancer Res 2004, 6:E1 (DOI 10.1186/bcr735) \\ (c) 2004 BioMed Central Ltd (Print ISSN 1465-5411; Online ISSN 1465-542X)
}

\begin{abstract}
The four-day biennial 8th Nottingham Breast Cancer Conference held at the East Midlands Conference Centre, University of Nottingham, UK (16-19 September 2003) once again proved to be a successful event. Recent advances in clinical and scientific research were presented to an international audience, covering a broad spectrum of breast cancer issues including prediction, diagnosis and treatment. Delegates were encouraged to participate in workshop sessions, which allowed the comprehensive discussion of existing and promising future advances in breast cancer care.
\end{abstract}

Keywords: breast cancer therapy, growth factors, oestrogen receptor, patient consent, prognosis

\section{Introduction}

The 8th Nottingham International Breast Cancer Conference was targeted towards scientists and healthcare professionals involved in breast cancer treatment and research. The meeting attracted about 900 delegates comprising surgeons, oncologists, radiologists, pathologists, scientists, and breast care and research nurses from both Europe and worldwide. Abstracts of submitted papers were published in the European Journal of Cancer [1].

\section{Endocrine - biology and clinical}

A comprehensive overview of the functional role of oestrogen receptor $\beta(E R \beta)$ in breast cancer was provided by Professor J- $\AA$ Gustavson from Sweden. Since its initial cloning in 1996, the precise role of ER $\beta$ in breast cancer remains ambiguous. Data were presented to suggest an anti-proliferative role of ER $\beta$ in protecting against abnormal cellular proliferation and cancer development in several tissue types including breast and prostate. The use of an ER $\beta$ knockout mouse model additionally implies a functional role of the receptor subtype in the maintenance of tissue integrity. Mice deficient in ER $\beta$ protein display a decreased expression of the basement membrane components collagen and laminin, suggesting an anti-metastatic role for ER $\beta$. The putative anti-proliferative function of ER $\beta$ was further reiterated by $\operatorname{Dr} A$ Shaaban (University of Liverpool and Clatterbridge Cancer Research Trust, UK). By using immunohistochemical staining techniques a progressive loss of ER $\beta$ protein was demonstrated from breast hyperplasia through to ductal carcinoma in situ (DCIS) and invasive cancer in comparison with normal tissue. The potential epigenetic mechanisms accounting for ER loss during breast cancer progression were discussed in presentations by $\mathrm{Dr} F$ Cooke (Mater Misericordiae Hospital, Dublin, Ireland) and Dr V Speirs (University of Leeds, UK). The technique of methylation-specific polymerase chain reaction (MSP) is a method of reliably identifying ER transcriptional silencing occurring as a result of promoter hypermethylation. Concordances of $61 \%$ and $65 \%$ were reported between MSP profiles and immunohistochemical staining for ER $\alpha$ and

ATAC = Arimidex, tamoxifen, alone or in combination; CGH = comparative genomic hybridisation; CMF = phosphamide, methotrexate and 5-fluorouracil; DCIS = ductal carcinoma in situ; $\mathrm{ER}=$ oestrogen receptor; HER = human endothelial growth factor receptor; $\mathrm{MSP}=$ methylation-specific PCR; VEGF = vascular endothelial growth factor. 
ER $\beta$, respectively. The data suggest potential applications of this technique in the prediction of patient response to adjuvant endocrine therapy and in the identification of resistant tumours.

\section{Future of endocrine treatment of breast cancer}

The future of endocrine treatment for breast cancer is currently uncertain since the interim analysis of the Arimidex, tamoxifen, alone or in combination (ATAC) trial [2]. The third-generation aromatase inhibitors are currently challenging the supremacy of tamoxifen as the 'gold standard' treatment for post-menopausal breast cancer. With a median follow-up of 47 months, the ATAC trial data continues to demonstrate superiority for anastrazole in terms of disease-free survival and the development of contralateral breast cancer [3]. Plans are currently in progress to produce tissue microarrays from the tumours excised from patients treated in the ATAC trial. This would subsequently permit the evaluation of the various markers associated with tumour recurrence and response to the therapies used within the ATAC trial. To illustrate, human endothelial growth factor receptor 2 (HER2) and other cell-surface growth factor receptors are proposed to cross-talk with the ER via pathways involving mitogen-activated protein kinase. Evidence exists that tumours overexpressing HER2 are resistant to tamoxifen but might be more sensitive to the inhibitory effects of anastrazole. The use of an ATAC tissue microarray would allow the investigation of this hypothesis.

Randomised clinical trials have previously shown the pure anti-oestrogen Faslodex to be as effective as anastrazole in reducing time to progression in patients with advanced breast cancer. New data were presented showing equivalent overall survival in patients treated with Faslodex in comparison with anastrazole. Faslodex therefore represents a useful additional drug for the treatment of patients with advanced disease. Questions still remain about the exact sequencing of treatment in advanced breast cancer. After relapse while taking tamoxifen, patients can be treated with either Faslodex or an aromatase inhibitor such as exemestane. However, Faslodex might offer an additional therapy after the failure of aromatase inhibitors.

\section{Predicting and targeting in breast cancer}

The future of breast cancer prognostication was explored in a lecture by the Dutch radiation oncologist $\mathrm{Dr} \mathrm{H}$ Bartelink. It is currently acknowledged that a proportion of breast cancer patients receive excessive treatment, whereas others remain vulnerable to local recurrence or relapse and might benefit from more intensive therapy. $\mathrm{Dr}$ Bartelink discussed the potential role of DNA microarray analysis in the prediction of tumour response to radiotherapy, chemotherapy, endocrine agents and other potential drugs. Data were presented from an expression array
70 genes that predict for the onset of distant metastases within 5 years. These include several genes encoding proteins involved in cell cycle progression, invasion and angiogenesis. Comparative genomic hybridisation (CGH) microarray data presented by $\operatorname{Dr} C$ Witton (Royal Infirmary Glasgow, UK, and Vysis Inc, Downers Grove, IL, USA) similarly characterised several genes putatively involved in breast cancer development. DNA from 100 breast tumour specimens was retrospectively analysed to reveal the amplification of genes involved in cell cycle progression and survival, including CCND1, AKT1, INSR, AKT2 and $E G F R$. This technology might prove clinically useful in the prediction of disease progression and patient response to new and existing therapies. The banking of tissue specimens from prospective randomised trials might additionally yield valuable information about the specific genes associated with response to various treatments.

\section{Tissue retention and patient consent issues}

Translational research is essential for both an increased understanding of the mechanisms of breast carcinogenesis and the development of new therapeutic strategies. After the Alder Hay debacle in 2000, the issue of tissue retention and banking has remained in the spotlight, potentially threatening translational research. These concerns were addressed in a workshop entitled 'EUROPA DONNA - issues surrounding the retention of tissues for breast cancer research'. EUROPA DONNA (http://www. cancerworld.org/progetti/cancerworld/europadonna/pagine/ home/homeframeeudonna.html) is a women's breast cancer advocacy organisation representing the views of women from 27 European member states, drawing attention to the need for improved breast cancer services and research.

In the UK since Alder Hay, most concerns about tissue retention have originated from the government and professional medical organisations; little regard has been given to the opinions of the breast cancer patient. This has been addressed by four independent groups within the UK: first, a consortium representing Addenbrooke's Hospital, Cambridge, and the Universities of Leicester and Nottingham; second, the Institute of Cancer Research and Royal Marsden Hospital, Sutton, for the Trial Management Groups and Trial Steering Committees for START (Standardisation of Radiotherapy) and TACT (Taxotere as Adjuvant Chemotherapy); third, Nottingham City Hospital; and fourth, the Leeds Teaching Hospitals NHS Trust. The first three groups used questionnaires to solicit the views of breast cancer patients undergoing treatment on the issue of tissue retention, whereas the last used a focus group consisting of breast cancer patients and 'non-threatening' female medical staff. Dr K Burnet (Addenbrooke's Hospital, University of Leicester, and University of Nottingham, UK) reported the outcome of a survey of 96 breast cancer patients questioned about their views on entry into clinical 
studies. Provided that the studies were fully explained in lay terms, about three-quarters of patients were willing to participate in multiple studies. Data presented by $\mathrm{Dr} \mathrm{L}$ Johnson (Institute for Cancer Research and Royal Marsden Hospital, Sutton, for the Trial Management Groups and Trial Steering Committees for START and TACT, UK) similarly reported a respective $81.1 \%$ and $98.4 \%$ of breast cancer patients to be willing to donate blood and paraffin-embedded tumour samples for use in medical research. The four studies therefore concluded that the vast majority of patients have no objection to the use of their reserve tissue for research purposes. It is evident from these studies that decisions on tissue retention should be made through consultation with the patients involved.

\section{Bone health in breast cancer}

The use of bisphosphonates in breast cancer was reviewed in a presentation by $\mathrm{Dr} \mathrm{R}$ Leonard. It was concluded that bisphosphonates do have a defined role in the treatment of both hypercalcaemia of malignancy and bone metastasis, acting to prevent pain and reduce the risk of fracture. Still to be established is the adjuvant role of bisphosphonates in the prevention of bone metastases and osteoporosis in breast cancer patients treated with aromatase inhibitors. This should be given precedence in view of the preliminary ATAC results. The AZURE (does adjuvant zoledronic acid reduce recurrence in patients with high risk localised breast cancer) and ZFAST (Zometa-Femara Adjuvant Synergy Trial) trials investigating the adjuvant use of zoledronic acid seek to provide answers to these questions, and the results will be of potential clinical importance.

\section{Goserelin in the adjuvant treatment of breast cancer}

An update of the Zoladex Early Breast Cancer Research Association (ZEBRA) trial was presented by Professor $\mathrm{R}$ Blamey (Nottingham City Hospital, UK). The trial has demonstrated equivalent disease-free and overall survival periods with the anti-androgen goserelin (Zoladex) to those achieved with standard cyclophospamide, methotrexate and 5-fluorouracil (CMF) therapy. Goserelin was found to be better tolerated than CMF by most patients, suggesting goserelin as an effective alternative to adjuvant CMF chemotherapy in premenopausal and perimenopausal patients with ER-positive and node-positive early stage breast cancers.

Results were presented by the Austrian Breast and Colorectal Cancer Study Group comparing the combined use of goserelin and tamoxifen as an alternative to standard CMF chemotherapy. A statistically significant improvement in disease-free and recurrence-free survival was observed in premenopausal patients with hormone-sensitive stage I/II tumours after the use of goserelin and tamoxifen as adjuvant therapy. These results were concurrent with the findings of the Italian Breast Cancer Study Group (National Cancer Research Institute, Genoa, Italy). Despite the encouraging evidence from randomised controlled trials, goserelin is rarely offered as an adjuvant treatment to eligible premenopausal breast cancer patients. This issue should be addressed.

\section{Breast cancer risk}

Dr G Evans (St Mary's Hospital and Withington Hospital, Manchester, UK) presented an evaluation of the various risk assessment models currently used in the clinic. Three models in common use historically (Gail, Claus and Ford) were compared with a more recent model developed by Tyrer and Cuzick. A failure of the older models is the underestimation of actual risk for women with a family history of breast cancer, suggesting that the Tyrer-Cuzick model is more accurate in this group of patients. On behalf of the Irish Breast Screening Programme, Dr S Boran (Mater Misericordiae Hospital, Dublin, Ireland) presented encouraging evidence from the newly established programme for users of hormone replacement therapy. Although such users are more likely to be recalled for extra mammography views or biopsy, the actual rates of breast cancer detection are not increased and those tumours detected are generally low grade and node negative.

\section{Diagnosis and prognosis}

A series of presentations addressing the diagnosis of breast disease was attended by approximately 150 delegates. Dr K Buhagiar (Western General Hospital, Edinburgh, UK) outlined proposals of an electronic proforma referral letter from general practitioner to specialist, with the aim of decreasing unnecessary delays in diagnosis. However, a study investigating the causes of a delay in breast cancer diagnosis of greater than 2 months identified several responsible factors such as inadequate imaging, lack of continuity of care and misinterpretation of results, as opposed to delays from primary care. Errors in the interpretation of screening mammograms can be avoided by the arbitration of disparate double readings of the films.

Both fine needle aspiration cytology and core biopsy are useful tools in the investigation of breast lesions. It was reported by Dr A Jah (Luton and Dunstable Hospital, UK) that the combined use of these two tests resulted in increases in sensitivities for screen-detected and symptomatic patients to $80.9 \%$ and $89.1 \%$, respectively. It was therefore recommended that patients should undergo both investigations during diagnosis, although this will undoubtedly incur additional expenditure. Core biopsy results cannot be processed in sufficient time to provide diagnosis at one-stop breast clinics. As presented by $\mathrm{Dr}$ M Klevesath (West Suffolk Hospital, Bury St Edmonds, UK) the use of touch imprint cytology of core biopsy specimens before formalin fixation is an accurate method of 
providing immediate results, with a sensitivity of $97 \%$ for predicting malignancy.

Dr G Wishart (Addenbrooke's Hospital, Cambridge, UK) compared the established Nottingham Prognostic Indicator score with the computer program 'Adjuvant!' [4], which is designed to assist in decision making about the use of adjuvant therapy. The use of this program gave an improved prognosis in 102 of 104 patients discussed at a 'mock' multidisciplinary meeting. The clinical implication for this is that 10 extra patients would not have been offered chemotherapy, and a further 14 patients predicted to have marginal benefits would have been given the option to decide treatment for themselves. As many as 24 patients could potentially have been spared chemotherapy. Although it was argued that Adjuvant! provides a more individual prognostic assessment, this scoring system does not take into account the method of detection and the fact that screen-detected tumours generally have a more favourable prognosis.

\section{Biology - growth factors}

The HER family (HER1-4) are transmembrane receptor tyrosine kinases that initiate epidermal growth factor (EGF) signalling pathways after ligand binding. The HER proteins are often aberrantly expressed in breast tumours. A presentation by Dr C Wong (Royal South Hants Hospital, Southampton, UK) compared the expression of HER2 in younger (19-35 years) and older (55-65 years) breast cancer patient groups. The expression of HER2 was found to be more prevalent in the younger patient cohort, inversely correlating with ER status. It was proposed that younger patients should be routinely tested for HER2 expression, to permit the modification of treatment regimens and the early use of Herceptin where appropriate. Serum and tissue concentrations of HER2 were compared in a study presented by Dr C Shaw (Mater Misericordiae Hospital, Dublin, Ireland). The analysis of samples from 151 breast cancer patients revealed no correlation between the HER2 concentration in serum and that in tissue. However, high concentrations of serum HER2 at the time of diagnosis were associated with high-grade disease, suggesting serum HER2 as a potential prognostic indicator. The potential mechanisms of growth inhibition by Herceptin were investigated by $\operatorname{Dr} \mathrm{C}$ McMonagle (University College Hospital, Galway, Ireland). Cell cycle arrest of HER2-positive breast cancer cells in response to Herceptin was correlated with the downregulation of $59 \%$ of the cell cycle genes involved in proliferation, as determined by complementary DNA microarray analysis.

Presentations by Dr S Tovey (Royal Infirmary, Glasgow, UK) and Dr G Boland (University Hospital of South Manchester, UK) discussed the putative role of HER1-4 in breast cancer. The expression of HER1-4 proteins in 55 primary breast tumours was compared with the prolif- eration rate, as determined by the incorporation of bromodeoxyuridine. A direct association was observed between HER1-3 positivity and a high rate of cellular proliferation, while HER4 expression was correlated with a low proliferation rate. In DCIS tumour specimens the expression of HER4 was associated with positive ER status and a low proliferation rate, suggesting HER4 as a favourable prognostic marker.

Scientific interest in angiogenic markers remains high, and circulating vascular endothelial growth factor (VEGF) concentrations are generally elevated in breast cancer patients. The angiogenic activity of VEGF is negatively regulated by the soluble receptor, termed sFlt-1. Concentrations of circulating VEGF and sFlt-1 were quantified in the serum of 51 patients with primary invasive breast cancer, as reported by Dr F Hoar (City Hospital, Birmingham, UK). The concentrations of VEGF were higher in the patient group than in healthy controls, while sFlt-1 concentrations remained similar in both groups, reinforcing the suggestion that the balance of pro-angiogenic and antiangiogenic factors is altered during malignant progression. Data presented by Dr D Morgan (Nottingham City Hospital, UK) similarly revealed a direct correlation between the high expression of VEGF-D in the primary tumour and an increased risk of local recurrence in young patients with breast cancer (below the age of 40 years). The potential clinical applications of a novel inhibitor of VEGF activity, termed ZD6474, were discussed by $\mathrm{Dr} S$ Wedge (AstraZeneca, Macclesfield, UK). Also inhibiting EGF signalling pathways, ZD6474 has been found to decrease blood vessel formation and tumour size in several human xenograft models including breast cancer. The compound is currently undergoing clinical evaluation in Phase II trials.

\section{Conclusion}

The meeting was conducted in an informal atmosphere, allowing lively discussion of the issues raised. Recent clinical and scientific advancements were highlighted, with particular relevance to diagnosis, prognostic indicators and novel therapeutic strategies. Data were presented from continuing clinical trials aimed at improving treatment and offering alternative therapies for premenopausal and postmenopausal patients with advanced and metastatic disease, including those who experience tumour recurrence while receiving 'gold standard' tamoxifen treatment. Promising advances in novel techniques such as tissue microarrays and $\mathrm{CGH}$ are set to revolutionise breast cancer diagnosis and prediction. These procedures will permit the more effective characterisation of tumour profiles before treatment, allowing the identification of drugresistant patients. Patient consent to the use of tumour samples for translational research is imperative for the successful improvement of breast cancer management and patient care in forthcoming years. 


\section{Competing interests}

None declared.

\section{References}

1. 8th Nottingham International Breast Cancer Conference, 16-18 September 2003: abstracts of submitted papers. Eur J Cancer 2003, Suppl 1.

2. Baum M, Budzar AU, Cuzick J, Forbes J, Houghton JH, Klijn JG, Sahmoud T; ATAC Trialists' Group: Anastrozole alone or in combination with tamoxifen versus tamoxifen alone for adjuvant treatment of postmenopausal women with early breast cancer: first results of the ATAC randomised trial. Lancet 2002, 359:2131-2139.

3. Buzdar A. Anastrozole as adjuvant therapy for early-stage breast cancer: implications of the ATAC trial. Clin Breast Cancer 2003, Suppl 1:S42-S48.

4. Ravdin PM, Siminoff LA, Davis GJ, Mercer MB, Hewlett J, Gerson $\mathrm{N}$, Parker HL: Computer program to assist in making early decisions about adjuvant therapy for women with early breast cancer. J Clin Oncol 2001, 19:980-991.

\section{Correspondence}

Jane L Limer, Molecular Medicine Unit, University of Leeds, St James's University Hospital, Leeds LS9 7TF, UK. Tel: +44 (0)113 206 5690;

fax: +44 (0)113244 4475; e-mail: j..l.limer@leeds.ac.uk 